\title{
Search for a dimuon resonance in the $\Upsilon$ mass region
}

\section{LHCD}

\section{The $\mathrm{LHCb}$ collaboration}

E-mail: martino.borsato@cern.ch

ABstract: A search is performed for a spin-0 boson, $\phi$, produced in proton-proton collisions at centre-of-mass energies of 7 and $8 \mathrm{TeV}$, using prompt $\phi \rightarrow \mu^{+} \mu^{-}$decays and a data sample corresponding to an integrated luminosity of approximately $3.0 \mathrm{fb}^{-1}$ collected with the $\mathrm{LHCb}$ detector. No evidence is found for a signal in the mass range from 5.5 to $15 \mathrm{GeV}$. Upper limits are placed on the product of the production cross-section and the branching fraction into the dimuon final state. The limits are comparable to the best existing over most of the mass region considered and are the first to be set near the $\Upsilon$ resonances.

Keywords: Beyond Standard Model, Hadron-Hadron scattering (experiments), Higgs physics

ARXiv EPrint: 1805.09820 


\section{Contents}

1 Introduction 1

2 Detector and simulation $\quad 2$

3 Event selection 3

4 Signal efficiencies $\quad 5$

5 Invariant mass fit $\quad 6$

6 Results 18

$\begin{array}{lll}7 & \text { Conclusions } & 10\end{array}$

$\begin{array}{ll}\text { A Results for other models } & 11\end{array}$

$\begin{array}{ll}\text { The LHCb collaboration } & 16\end{array}$

\section{Introduction}

The only known elementary spin- 0 particle is the resonance of mass $125 \mathrm{GeV}$ ( $c=1$ throughout this paper) discovered at the LHC, $H$, whose properties are found to be consistent with those of the Standard Model (SM) Higgs boson [1, 2]. However, additional spin-0 bosons, $\phi$, arise in many extensions of the SM and are often predicted to be lighter than the $H$ boson mass, $m(H)$ [3]. Examples of models with light (pseudo-)scalar particles are the next-to-minimal supersymmetric SM (NMSSM) [4-6], Little Higgs models [7-9] and the two-Higgs doublet model with an additional scalar [3]. Scalar fields can also provide portals to so-called dark sectors that are neutral under SM interactions and that might include dark matter particles [10-12]. A scalar portal mediated by a light particle can also be associated to the inflation of the early Universe $[13,14]$.

An extensive and diverse set of searches has been performed for new spin-0 particles with masses less than $m(H)$ (see ref. [15] for a recent review). Most searches performed by the ATLAS and CMS collaborations rely on the hypothetical decay $H \rightarrow \phi \phi$ and on the reconstruction of the two $\phi$ boson decays in the $\mu^{+} \mu^{-}, \tau^{+} \tau^{-}$and $b \bar{b}$ final states. A complementary strategy [15] consists of searching for the direct production of $\phi$ bosons in $p p$ collisions via, e.g. gluon-gluon fusion. Searches of this type performed at the LHC have aimed at reconstructing a possible $\phi$ boson in its decay to either $\gamma \gamma, \tau^{+} \tau^{-}$or $\mu^{+} \mu^{-}$. A recent search in the $\gamma \gamma$ final state [16] explored a mass range down to $m(\phi)=70 \mathrm{GeV}$, while one employing $\tau^{+} \tau^{-}$explored masses down to $m(\phi)=90 \mathrm{GeV}$ [17]. Masses as low 
as $m(\phi)=25 \mathrm{GeV}$ were also investigated in the $\phi \rightarrow \tau^{+} \tau^{-}$decay using the signature of a $\phi$ boson produced in association with two $b$ jets [18]. For lower masses, searches in the dimuon spectrum are currently the most sensitive [15] and include $\phi$ bosons produced in either gluon-gluon fusion in LHC collisions [19], $\Upsilon(1 S)$ radiative decays [20, 21] or rare $b$-hadron decays $[22,23]$.

As shown in ref. [24], the LHCb detector has good sensitivity to light spin-0 particles due to its high-precision spectrometer and its capability of triggering on objects with small transverse momenta. LHCb has already searched for prompt dark photons decaying to dimuons with invariant masses up to $70 \mathrm{GeV}$ [25] using $p p$ collisions at $13 \mathrm{TeV}$ corresponding to an integrated luminosity of $1.6 \mathrm{fb}^{-1}$. These results were recently reinterpreted in the context of a $\phi$ boson search and provide the best limits in the mass region between 10.6 to 70 $\mathrm{GeV}$ [15], even though this search was optimised for the dark photon production kinematics. However, all searches in $p p$ collisions exclude the region dominated by $\Upsilon$ resonances.

This article presents a search for a narrow dimuon resonance in the mass region between 5.5 and $15 \mathrm{GeV}$. The excellent mass resolution of the $\mathrm{LHCb}$ detector is exploited to study the region close to the $\Upsilon$ resonances that was not explored in previous searches. For this analysis, signal candidates are selected from $p p$ collision data corresponding to an integrated luminosity of $0.98(1.99) \mathrm{fb}^{-1}$, recorded with the LHCb detector during 2011 (2012) at a centre-of-mass energy of $\sqrt{s}=7$ (8) $\mathrm{TeV}$ (a data set statistically independent from that of ref. [25]).

The results are interpreted in the context of a $\phi$ boson produced directly in the $p p$ collision through gluon-gluon fusion. The analysis has been designed in a model-independent way for any prompt dimuon resonance, be it predicted by the $\operatorname{SM}\left(e . g . \eta_{b} \rightarrow \mu^{+} \mu^{-}\right.$as suggested in ref. [24]) or not. In order to be independent of the production mechanism, the data set is analysed separately in bins of the dimuon kinematics and for the two collision energies. The results are also independent of the resonance spin, allowing for an interpretation in terms of a vector boson, $A^{\prime}$.

\section{Detector and simulation}

The LHCb detector $[26,27]$ is a single-arm forward spectrometer covering the pseudorapidity range $2<\eta<5$, designed for the study of particles containing $b$ or $c$ quarks. The detector includes a high-precision tracking system consisting of a silicon-strip vertex detector surrounding the $p p$ interaction region [28], a large-area silicon-strip detector located upstream of a dipole magnet with a bending power of about $4 \mathrm{Tm}$, and three stations of silicon-strip detectors and straw drift tubes [29] placed downstream of the magnet. The tracking system provides a measurement of momentum, $p$, of charged particles with a relative uncertainty that varies from $0.5 \%$ at low momentum to $1.0 \%$ at $200 \mathrm{GeV}$. The minimum distance of a track to a primary vertex (PV), the impact parameter (IP), is measured with a resolution of $\left(15+29 \mathrm{GeV} / p_{\mathrm{T}}\right) \mu \mathrm{m}$, where $p_{\mathrm{T}}$ is the component of the momentum transverse to the beam. Different types of charged hadrons are distinguished using information from two ring-imaging Cherenkov detectors [30]. Photons, electrons and hadrons are identified by a calorimeter system consisting of scintillating-pad (SPD) and 
preshower detectors, an electromagnetic calorimeter and a hadronic calorimeter. Muons are identified by a system composed of alternating layers of iron and multiwire proportional chambers [31].

The online event selection is performed by a trigger [32], which consists of a hardware stage, based on information from the calorimeter and muon systems, followed by a software stage, which applies a full event reconstruction. In this analysis, signal candidates are first required to pass the hardware trigger, which selects events containing at least one muon with $p_{\mathrm{T}}>1.5(1.8) \mathrm{GeV}$ in the $7(8) \mathrm{TeV}$ data sample. The subsequent software trigger requires events with either a muon with $p_{\mathrm{T}}>10 \mathrm{GeV}$, or alternatively, a pair of muons having an invariant mass larger than $4.7 \mathrm{GeV}$, forming a good quality vertex and with the larger muon $p_{\mathrm{T}}$ exceeding $4.8 \mathrm{GeV}$. A global event cut (GEC) is also applied at the hardware stage, which requires the number of hits in the SPD to be less than 600 .

In the simulation, $p p$ collisions are generated using PYTHIA $[33,34]$ with a specific LHCb configuration [35]. Decays of hadronic particles are described by EvTGEN [36], in which final-state radiation is generated using Pнотоs [37]. The interaction of the generated particles with the detector, and its response, are implemented using the GEANT4 toolkit $[38,39]$ as described in ref. [40].

\section{Event selection}

A dimuon candidate is formed using two oppositely charged tracks identified as muons, which must satisfy the requirements of the hardware and software stages of the trigger. The vertex formed by the two tracks is required to be of good quality and to be consistent with the location of the primary vertex. Finally, the reconstructed proper decay time is required to be less than 0.1 ps to suppress background from muons produced in the decays of heavy flavour hadrons.

The dimuon invariant mass spectrum is investigated in the range from $5.5 \mathrm{GeV}$, above the region dominated by $b$-hadron decays, up to $15 \mathrm{GeV}$. In this mass region the $m(\phi)$ resolution is about $0.5 \%$ and the total acceptance for $\phi$ bosons produced via gluon-gluon fusion is between 2 and $3 \%$.

A fiducial region is defined for the kinematics of the dimuon candidate: each muon is required to be within $2.0<\eta<4.9$, and the higher (lower) muon $p_{\mathrm{T}}$ is required to be in excess of $4.8 \mathrm{GeV}(2.5 \mathrm{GeV})$. Moreover, the $\phi$ boson candidate $p_{\mathrm{T}}$ is required to be between 7.5 and $50 \mathrm{GeV}$ and its pseudorapidity between 2 and 4.5. The search is then performed in 6 bins of $p_{\mathrm{T}}(\phi)$ and 2 bins of $\eta(\phi)$ as well as separately for the two $p p$ collision energies, for a total of 24 separate samples. As shown in figure 1, the binned analysis provides better separation of signal from background if the $\phi$ boson production spectrum is significantly different from that of the background dimuon candidates.

Apart from the narrow $\Upsilon(n S)(n=1,2,3)$ resonances, the selected candidates are composed of three categories: genuine muon pairs produced via the Drell-Yan mechanism, pairs of displaced muons coming from heavy flavour decays, and wrong associations of one such muon with a prompt pion that is misidentified as a muon. While the DrellYan component is indistinguishable from a signal with the same production spectrum, the 


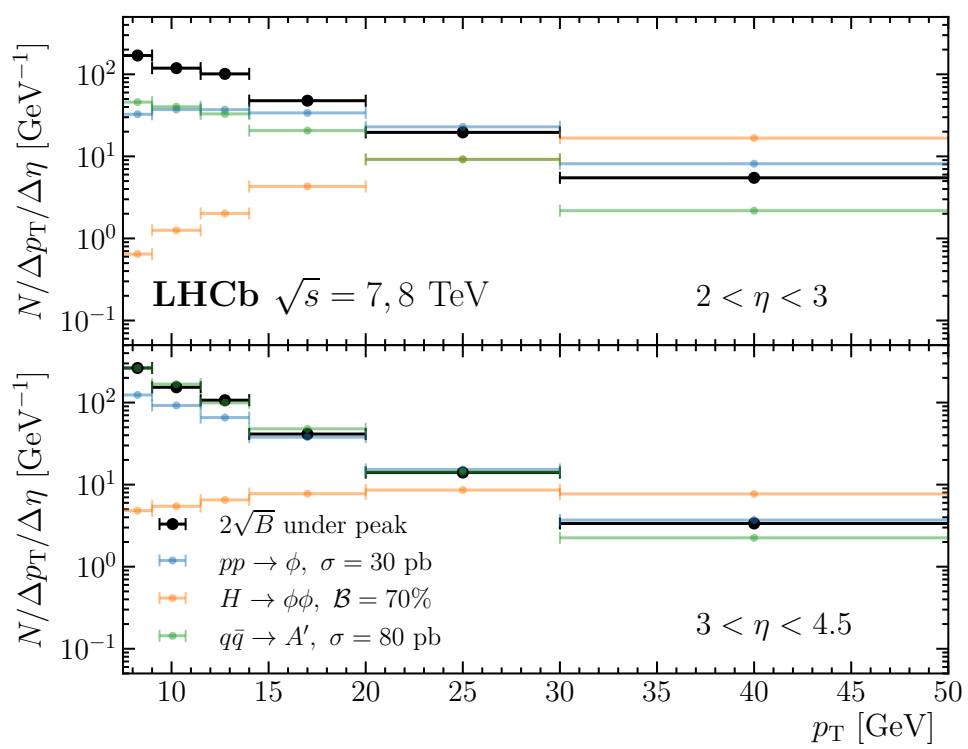

Figure 1. The expected sensitivity, defined as $2 \sqrt{B}$, where $B$ is the background under a dimuon peak with invariant mass $11 \mathrm{GeV}$, is shown for the $12\left[p_{\mathrm{T}}, \eta\right]$ bins. For comparison, signal yields in the various bins are shown for three different production mechanisms: a $\phi$ boson produced via gluon-gluon fusion, a $\phi$ boson coming from a $H \rightarrow \phi \phi$ decay and a vector $A^{\prime}$ boson produced via the Drell-Yan mechanism.

other two categories can be reduced. For this purpose, a multivariate (MVA) classifier based on the uniform boosting (uBoost) algorithm [41] is used, where a boosted decision tree is trained to separate signal from background candidates. This technique has been successfully used in previous LHCb searches [22], as it avoids biasing the mass spectrum and, most importantly, it simplifies the determination of the classification efficiency, which can be evaluated for a single mass using, for example, $\Upsilon(1 S)$ data. The MVA classifier is trained using a signal sample consisting of simulated Drell-Yan events and a background data sample composed of pairs of muon candidates with the same electric charge.

The classifier is trained on the following kinematic and topological features: IP, $p_{\mathrm{T}}$, momentum and track-fit $\chi^{2}$ of each muon candidate; minimum IP $\chi^{2}$ of both muons with respect to any PV in the event, where the IP $\chi^{2}$ is defined as the difference between the vertex-fit $\chi^{2}$ of a PV reconstructed with and without the muon; the angle between the positive muon in the $\phi$ boson rest frame and the direction opposite to that of the $\phi$ boson in the laboratory frame; IP of the dimuon candidate; and the isolation variable defined in ref. [42], related to the number of good two-track vertices a muon can make with other tracks in the event, to reduce the background from heavy flavour decays.

In order to account for small differences between simulation and data, a correction is applied through a multi-dimensional weighting [43]. This correction is determined by matching simulation and data in various detector-related variables of a $\Upsilon(1 S)$ sample. Examples of the variables showing discrepancies are the track-fit $\chi^{2}$ and the IP $\chi^{2}$ of the muons. For the data sample, background is statistically subtracted using the sPlot technique [44] based on a fit to the $\Upsilon(1 S)$ dimuon mass peak. 
To determine the best MVA requirement, the ratio $\epsilon_{S} /(3 / 2+\sqrt{B})$ [45] is maximised, where $\epsilon_{S}$ is the signal efficiency and $B$ the mean background yield. For this, $\epsilon_{S}$ is taken from $p p \rightarrow \phi \rightarrow \mu^{+} \mu^{-}$simulated samples, while an estimate of the average background yield under the hypothetical signal peak is taken from the mass sidebands of the $\Upsilon(n S)$ region in data. The resulting MVA requirement is about $90 \%$ efficient on reconstructed $p p \rightarrow \phi \rightarrow \mu^{+} \mu^{-}$signal while it reduces the background by about $40 \%$. By comparing the samples composed of same-sign and opposite-sign muons, the genuine dimuon purity is estimated to be about $50 \%$.

\section{Signal efficiencies}

The determination of signal efficiencies relies on simulated dimuon samples, which are corrected for small inaccuracies of the simulation using control data samples.

Trigger efficiencies are above $90 \%$. They are determined from simulation and checked on data. The efficiency of the global event cut is instead taken from data using a sample of $\Upsilon(1 S)$ candidates selected in the hardware trigger using a much looser requirement on the SPD multiplicity. Given the event multiplicity does not significantly change with dimuon mass, the same GEC efficiency is used for the whole range of masses.

The reconstruction and selection efficiencies are determined using simulation. The muon track reconstruction efficiency is corrected as a function of the track kinematics using a data sample of $J / \psi \rightarrow \mu^{+} \mu^{-}$decays [46]. The total systematic uncertainty related to this procedure is about $0.8 \%$.

The efficiency of the MVA selection is computed using the weighted simulation sample and is tested on $\Upsilon(1 S)$ candidates selected without applying the MVA criterion. The efficiency difference in each bin is below $2 \%$, which is assigned as a systematic uncertainty. The MVA response is decorrelated from the dimuon mass due to the use of the uBoost technique allowing this cross-check to be valid for the whole range of $m(\phi)$ considered.

The muon identification efficiency is calculated using a sample of $J / \psi \rightarrow \mu^{+} \mu^{-}$decays, following the procedure in ref. [47]. In addition to the statistical uncertainty due to the finite size of the calibration sample, a systematic uncertainty between 1 and $7 \%$ is assigned due to the finite width of the kinematic bins used.

Finally, the total efficiency in each bin is obtained as the product of the efficiencies described above, where different sources of systematic uncertainties are assumed to be fully correlated. Efficiencies for the $12\left[p_{\mathrm{T}}, \eta\right]$ bins and for three different $m(\phi)$ values are shown in figure 2 for the $8 \mathrm{TeV}$ sample. Due to the fiducial region defined, the separation in kinematic bins and the use of the uBoost technique, the efficiencies are minimally correlated with the $\phi$ boson mass. A quadratic function is also fitted to the efficiency mass dependence and compared to the mass average. The mean value between the two efficiencies is taken as the nominal value while the difference is assigned as a systematic uncertainty. The dependence of the efficiency on the $\phi$ boson kinematics due to the $\left[p_{\mathrm{T}}, \eta\right]$ bin size is evaluated by comparing the efficiencies in each bin obtained for $p p \rightarrow \phi$ production to those obtained for a $\phi$ boson originating from the decay $H \rightarrow \phi \phi$. The latter production mode gives a vastly different spectrum, with larger $p_{\mathrm{T}}$ and a more central $\eta$ distribution, as shown in 


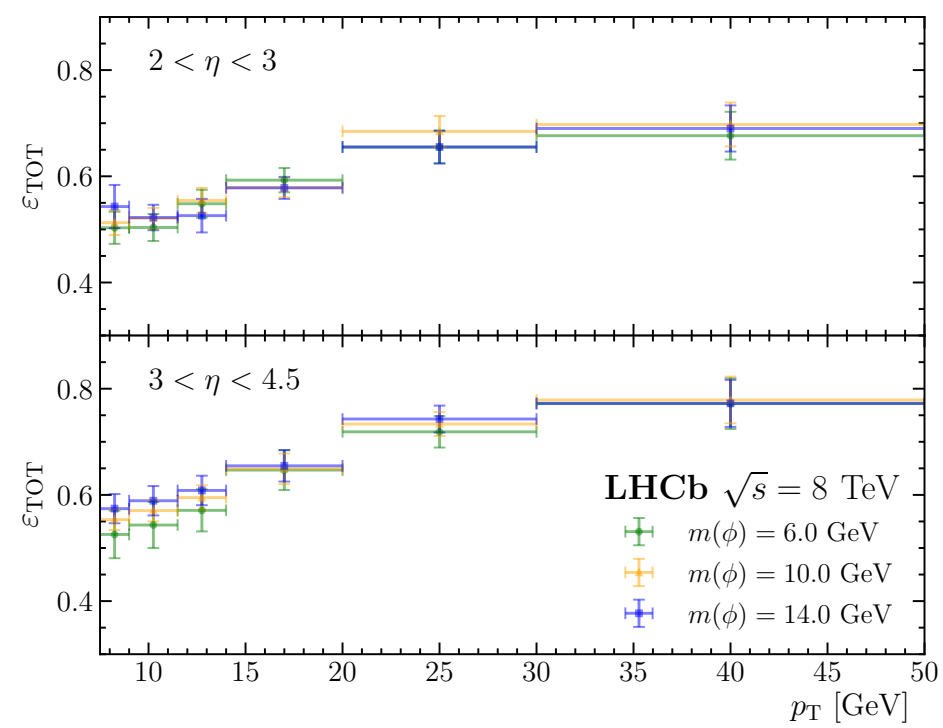

Figure 2. Total efficiency as a function of the $p_{\mathrm{T}}$ of the dimuon candidate for the two $\eta(\phi)$ bins considered, obtained for three different $\phi$ boson mass hypotheses.

figure 1. The small differences (1-5\%) in the efficiencies found are assigned as systematic uncertainties.

For the case where the boson is a vector, a systematic uncertainty of less than $5 \%$ is assigned to account for the dependency of the total efficiency on the boson polarisation. It is evaluated by weighting the spin- $0 \phi$ boson sample to match the angular distribution of a vector boson with either longitudinal or transverse polarisation.

\section{Invariant mass fit}

The $\phi$ boson signal yield is determined for each mass value with fits to the full dimuon invariant mass spectrum. Due to their complexity, the fits are computed by parallelising the processes on a Graphics Processing Unit (GPU), for which the framework developed in ref. [48] is used, where the minimisation is based on Minuit [49]. The natural width of the $\phi$ boson candidate is assumed to be negligible compared to the detector mass resolution, which is $\sigma\left(m_{\mu \mu}\right) / m(\phi) \approx 0.5 \%$ [27]. These fits are performed simultaneously in the 12 production kinematic bins, sharing some of the parameters. The $\phi$ boson mass hypotheses are scanned in steps of $\sigma\left(m_{\mu \mu}\right) / 2$. The detector resolution on the dimuon mass is modelled according to $\eta, p_{\mathrm{T}}$ and $m(\phi)$. The resolution model is used to simultaneously fit the $\Upsilon(n S)$ peaks, which are used for its calibration. Furthermore, in order to increase the invariant mass region scanned and to get as close as possible to the $\Upsilon(n S)$ resonances, a precise modelling of the $\Upsilon(n S)$ mass-distribution tails is needed. For this purpose, the reconstructed dimuon mass, $m_{\mu \mu}$, is modelled by a Gaussian-smeared Hypatia distribution, $\mathcal{S}$, which is defined as

$$
\begin{aligned}
\mathcal{S}\left(m_{\mu \mu}, m(\phi), \sigma_{\mathrm{MS}}, \sigma_{\mathrm{SR}}, \lambda, \beta, a, n\right)= & \frac{1}{\sigma_{\mathrm{MS}}} e^{-\frac{1}{2}\left(\frac{m_{\mu \mu}-m(\phi)}{\sigma_{\mathrm{MS}}}\right)^{2}} \\
& \otimes \mathcal{I}\left(m_{\mu \mu}, m(\phi), \sigma_{\mathrm{SR}}, \lambda, \zeta \rightarrow 0, \beta, a, n\right),
\end{aligned}
$$


where $\mathcal{I}$ is the Hypatia function [50], a generalised Crystal Ball (CB) [51] with a hyperbolic core that gives an excellent description of non-Gaussian tails, given by

$$
\begin{aligned}
& \mathcal{I}\left(m_{\mu \mu}, m(\phi), \sigma_{\mathrm{SR}}, \lambda, \zeta, \beta, a, n\right) \\
& \quad \propto \begin{cases}G\left(m(\phi)-a \sigma_{\mathrm{SR}}\right) & \text { if } \frac{m_{\mu \mu}-m(\phi)}{\sigma_{\mathrm{SR}}}>-a, \\
G\left(m(\phi)-a \sigma_{\mathrm{SR}}\right)\left(1-m_{\mu \mu} /\left(n \frac{G\left(m(\phi)-a \sigma_{\mathrm{SR}}\right)}{G^{\prime}\left(m(\phi)-a \sigma_{\mathrm{SR}}\right)}-a \sigma_{\mathrm{SR}}\right)\right)^{-n} & \text { otherwise, }\end{cases}
\end{aligned}
$$

and $G(x) \equiv G\left(x, m(\phi), \sigma_{\mathrm{SR}}, \lambda, \zeta, \beta\right)$ is its core, defined as

$$
\begin{aligned}
& G\left(x ; m(\phi), \sigma_{\mathrm{SR}}, \lambda, \zeta, \beta\right) \propto \\
& \quad\left((x-m(\phi))^{2}+A_{\lambda}^{2}(\zeta) \sigma_{\mathrm{SR}}^{2}\right)^{\frac{1}{2} \lambda-\frac{1}{4}} e^{\beta(x-m(\phi))} K_{\lambda-\frac{1}{2}}\left(\zeta \sqrt{1+\left(\frac{x-m(\phi)}{A_{\lambda}(\zeta) \sigma_{\mathrm{SR}}}\right)^{2}}\right),
\end{aligned}
$$

where $G^{\prime}$ is the derivative of $G$ (defined in eq. 5.3), $K_{\lambda}$ are the cylindrical harmonics or special Bessel functions of the third kind, $\beta$ is the asymmetry of the core, $a$ and $n$ are CB-like radiative-tail parameters, and $A_{\lambda}^{2}=\zeta K_{\lambda}(\zeta) / K_{\lambda+1}(\zeta)$. The parameter $\zeta$ is known to be small in most cases [50], and thus, is fixed to an arbitrarily small value. In order to reduce the number of free parameters in the simultaneous fits, a parametrisation of the dependence of the parameters defined above on $p_{\mathrm{T}}, \eta$ and $m(\phi)$ is obtained from the simulation. The parameters $\beta, n$ and $a$ are found to be independent of $p_{\mathrm{T}}, \eta$ and $m(\phi)$. The parameter $n$ is fixed to the value obtained from the simulation, while $\beta$ and $a$ are shared among different kinematic bins and mass hypotheses in the fit. Further information about these functions and their parameters can be found in ref. [50].

The Gaussian smearing factorises the mass resolution model into two components: the multiple scattering (MS) information, which is encoded in the smearing parameter $\sigma_{\mathrm{MS}}$; and the spatial resolution information, which is given by $\sigma_{\mathrm{SR}}$ and $\lambda$. In this parametrisation the value of $\sigma_{\mathrm{MS}}$ can be fixed from the ramp-up of the mass-error distribution without increasing the dimensionality of the fit. The mass error is obtained in the vertex fit and the ramp-up position is defined as the mass error corresponding to the fifth percentile of the distribution. The parameter $\sigma_{\mathrm{MS}}$ depends on the kinematics, and thus, is modelled in bins of $p_{\mathrm{T}}, \eta$ and $m(\phi)$ on the continuum background. The $m(\phi)$ dependence of this MS parameter is studied in bins of dimuon mass and is modelled by a linear fit. The $\sigma_{\mathrm{MS}}$ parameter in data is found to be in excellent agreement with the simulation, and therefore, no systematic uncertainty is assigned.

The continuous dimuon background is modelled with an exponential function multiplying Legendre polynomials, $P_{k}$, up to order $N$. The background shape parameters and yields are fit separately in each $\left[p_{\mathrm{T}}, \eta\right]$ bin. For each $m(\phi)$, the model has to describe the background under the signal peak, $B$, to a precision exceeding its expected statistical fluctuation, $\sqrt{B}$. The background model is tested on a sample composed of simulated Drell-Yan dimuon events and same-sign dimuon data events. The same-sign dimuon mass 
spectrum is expected to be representative of the background coming from pions misidentified as muons. In this mass spectrum, the candidate fit model is required to describe any structure with a width larger than $4 \sigma\left(m_{\mu \mu}\right)$ to a precision better than $0.5 \sqrt{B}$. Furthermore, a similar test is performed on a large simulated sample of muon pairs coming from heavy-flavour decays. This background component is expected to give narrower structures, therefore the above requirement is reduced to $0.3 \sqrt{B}$. These requirements are well satisfied by a background model with an exponential function multiplied by Legendre polynomials of order $N=10$, which is taken as reference.

The results of the fit to data in the whole mass region is shown in figure 3 , where all kinematic bins have been combined. The figure also shows how different $\phi$ boson mass peaks would look like.

The resolution function has 17 free parameters. The fits for $\phi$ boson mass hypotheses far from the $\Upsilon(n S)$ peaks are found to be largely independent of the signal model. However, for $m(\phi)$ close to the $\Upsilon(n S)$ resonances, the estimate of the background under a possible $\phi$ boson peak depends on the precise modelling of the $\Upsilon(n S)$ tails. In particular, significant differences are observed using a resolution function with fewer assumptions on the kinematic dependence of $\beta, a$ and $\lambda$. The $m(\phi)$ hypotheses for which the two background estimations differ with a significance larger than one standard deviation in any kinematic bin are not considered in the $\phi$ boson search. In addition, any $m(\phi)$ hypothesis where the fit gives a correlation between the signal yield and any of the $\Upsilon(n S)$ yields in excess of $20 \%$, is also excluded from the search.

\section{Results}

The fit results are found to be compatible with the background-only hypothesis. Upper limits at $95 \%$ Confidence Level (CL) are set on spin- $0 \phi$ bosons produced directly from the $p p$ collision. Pseudoexperiments are generated based on the fitted background probability distribution functions and upper limits are determined using the CLs approach [52, 53]. Measured integrated luminosities, simulated signal production spectra and the modelindependent efficiencies given in section 4 are used to compute expected signal yields in each $\left[p_{\mathrm{T}}, \eta\right]$ bin. Systematic and statistical uncertainties on the efficiency are propagated to the limit calculation, summing them in quadrature and taking into account their correlations among different bins. The integrated luminosities for the 7 and $8 \mathrm{TeV}$ samples are measured [54] with a precision of $1.7 \%$ and $1.2 \%$, respectively.

The production kinematics for spin- $0 \phi$ bosons are simulated using the MSSM pseudoscalar production implemented in PYтніA 8 [33]. Gluon-gluon fusion dominates, contributing more than $90 \%$ to the production cross-section in the whole $\phi$ boson mass range. In order to set limits on new spin-0 particles in terms of couplings, interference effects with spin-0 bottomonium states should be considered [24], but this is beyond the scope of this analysis. Therefore, upper limits are set on the product of the production cross-section and the dimuon branching fraction, $\sigma(p p \rightarrow \phi) \times \mathcal{B}\left(\phi \rightarrow \mu^{+} \mu^{-}\right)$. Since the cross-section depends on the collision energy, the limits are set for $\sqrt{s}=8 \mathrm{TeV}$ and the result from $7 \mathrm{TeV}$ is combined by taking the expected fraction of cross-sections as a function of $m(\phi)$, 

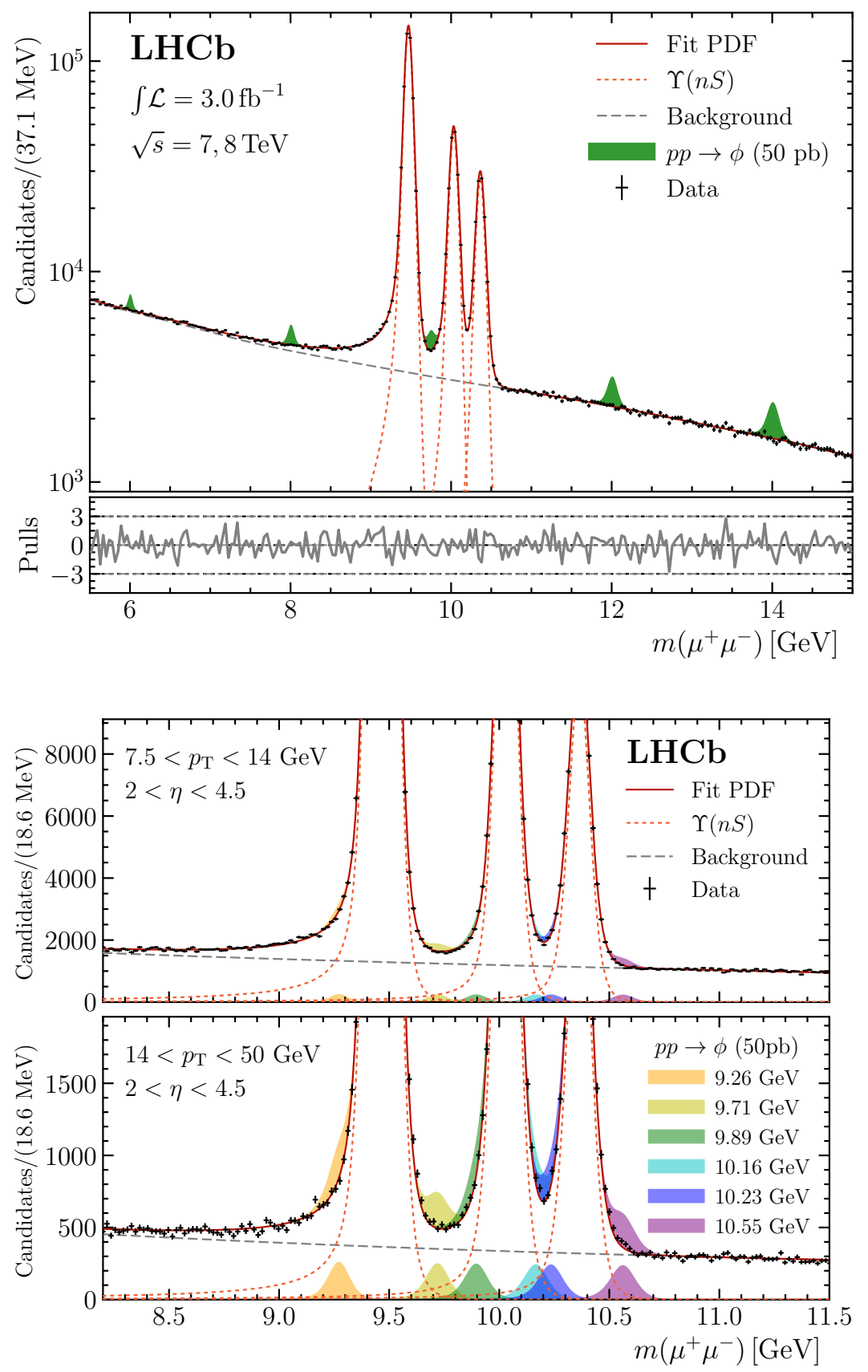

Figure 3. (Top) Fit to the dimuon invariant mass distribution in the whole scanned region. All $\left[p_{\mathrm{T}}, \eta\right]$ bins as well as the 7 and $8 \mathrm{TeV}$ data sets are combined. Peaks for five $\phi$ boson mass hypotheses are displayed in green, assuming $\sigma(p p \rightarrow \phi) \times \mathcal{B}\left(\phi \rightarrow \mu^{+} \mu^{-}\right)=50 \mathrm{pb}$. (Bottom) A closeup view of the mass spectrum in the $\Upsilon(n S)$ region together with $\phi$ boson mass peaks for the tested $m(\phi)$ values closest to the three $\Upsilon(n S)$ narrow resonances. To show how the $\Upsilon(n S)$ mass tails change with the kinematics, two regions of $p_{\mathrm{T}}$ are displayed in the plot. 


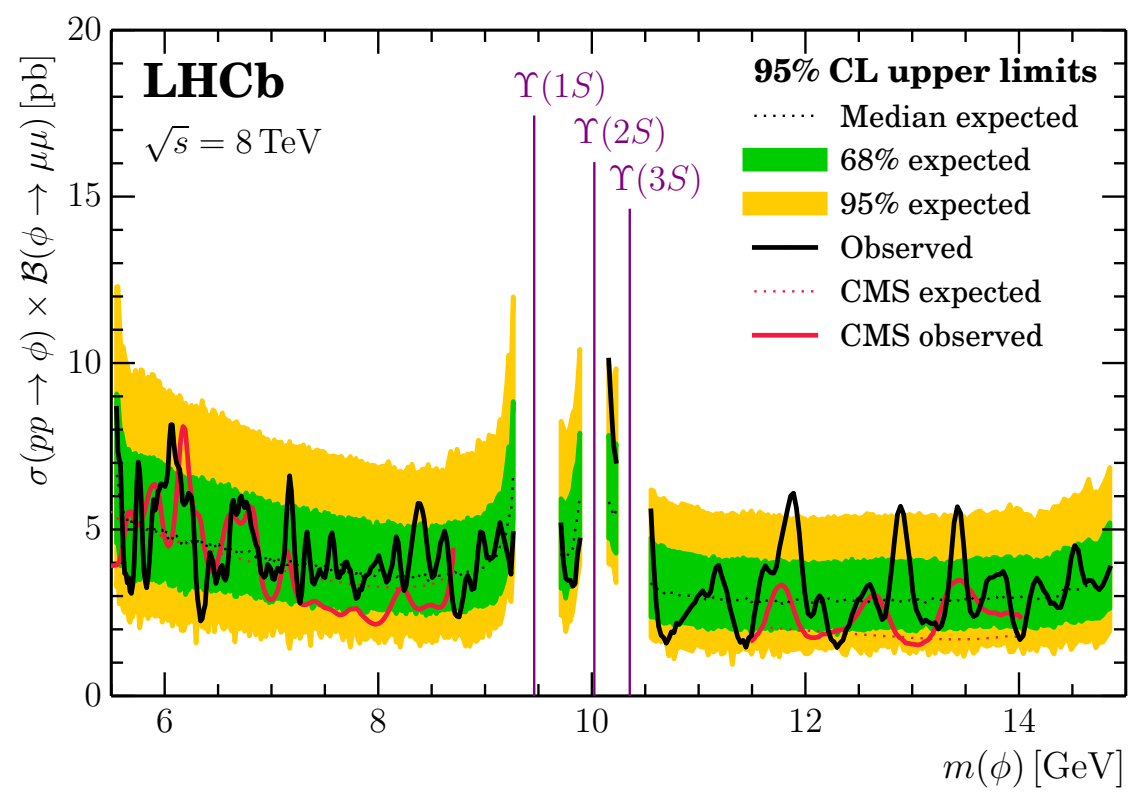

Figure 4. Upper limits on the direct production of a spin-0 boson decaying to $\mu^{+} \mu^{-}$in $8 \mathrm{TeV} p p$ collisions.

based on the framework detailed in ref. [24]. This ratio of cross-sections is roughly equal to the ratio of collision energies and has a small dependence on $m(\phi)$ of order $4 \%$ within the mass range considered. The observed limits are given in figure 4 along with the range of limits expected for the background-only hypothesis.

In appendix $\mathrm{A}$ the upper limits are interpreted for $\phi$ bosons coming from the decay of the $125 \mathrm{GeV}$ Higgs boson to two $\phi$ bosons and for vector $A^{\prime}$ bosons with Drell-Yan production. If the vector $A^{\prime}$ boson is interpreted as a dark photon, these are the first limits in the region between 9.1 and $10.6 \mathrm{GeV}$. Furthermore, reinterpretation of the limits in any other model involving the production of a dimuon resonance in the mass range considered is possible by using the information given in the supplemental material.

\section{Conclusions}

In summary, a search is presented for a hypothetical light dimuon resonance, produced in $p p$ collisions recorded by the $\mathrm{LHCb}$ detector at centre-of-mass energies of 7 and $8 \mathrm{TeV}$. A sample of dimuon candidates with invariant mass between 5.5 and $15 \mathrm{GeV}$ corresponding to an integrated luminosity of $3.0 \mathrm{fb}^{-1}$ is used. No evidence for a signal is observed and limits are placed on a benchmark model involving a new light spin-0 boson, $\phi$, decaying to a pair of muons. For the case in which the $\phi$ boson is produced directly in the $p p$ collision, the limits obtained are comparable with the best existing. Furthermore, by exploiting the excellent LHCb dimuon mass resolution and a detailed study of the $\Upsilon(n S)$ mass tails, limits are set in a previously unexplored range of $m(\phi)$ between 8.7 and $11.5 \mathrm{GeV}$. This search is designed to be largely model independent and tools are given in the supplemental material that allow for a simple reinterpretation of the result for different models. These 

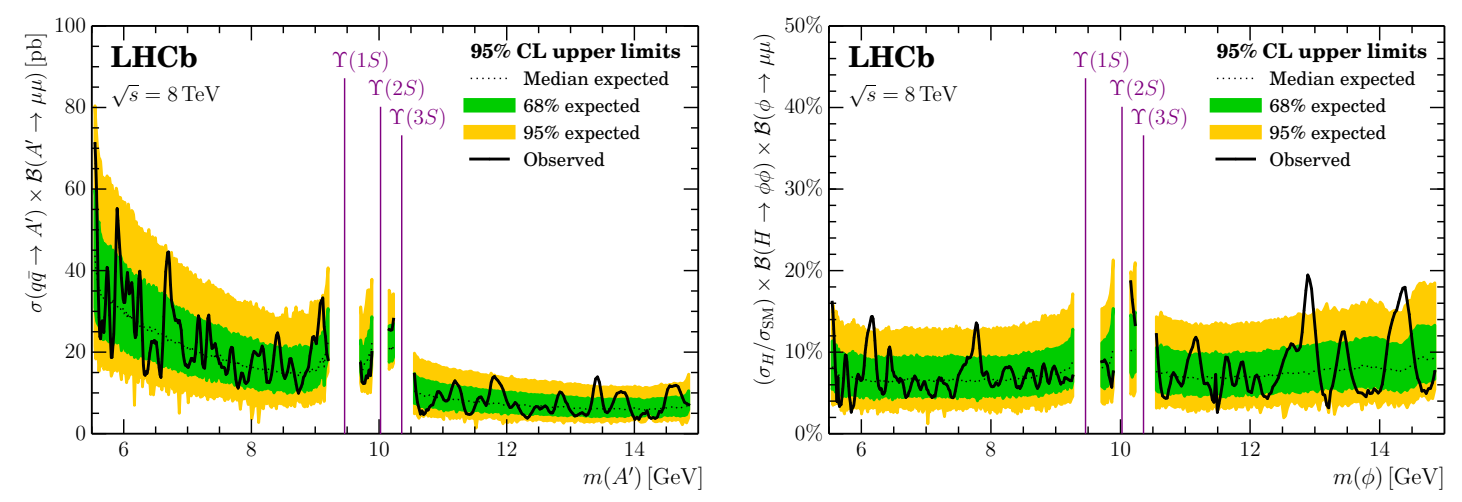

Figure 5. (Left) Upper limits on the production of vector $A^{\prime}$ bosons produced in $8 \mathrm{TeV} p p$-collisions through Drell-Yan and decaying to $\mu^{+} \mu^{-}$. (Right) Upper limits on the branching fraction of a SM Higgs decaying to two $\phi$ bosons followed by the decay of one of the two to $\mu^{+} \mu^{-}$.

results showcase the sensitivity of the LHCb experiment to light spin-0 bosons produced in $p p$ collisions and its capability of closing the gaps in the invariant mass distributions by means of a superior mass resolution.

\section{Acknowledgments}

We express our gratitude to our colleagues in the CERN accelerator departments for the excellent performance of the LHC. We thank the technical and administrative staff at the LHCb institutes. We acknowledge support from CERN and from the national agencies: CAPES, CNPq, FAPERJ and FINEP (Brazil); MOST and NSFC (China); CNRS/IN2P3 (France); BMBF, DFG and MPG (Germany); INFN (Italy); NWO (The Netherlands); MNiSW and NCN (Poland); MEN/IFA (Romania); MinES and FASO (Russia); MinECo (Spain); SNSF and SER (Switzerland); NASU (Ukraine); STFC (United Kingdom); NSF (U.S.A.). We acknowledge the computing resources that are provided by CERN, IN2P3 (France), KIT and DESY (Germany), INFN (Italy), SURF (The Netherlands), PIC (Spain), GridPP (United Kingdom), RRCKI and Yandex LLC (Russia), CSCS (Switzerland), IFIN-HH (Romania), CBPF (Brazil), PL-GRID (Poland) and OSC (U.S.A.). We are indebted to the communities behind the multiple open-source software packages on which we depend. Individual groups or members have received support from $\mathrm{AvH}$ Foundation (Germany), EPLANET, Marie Skłodowska-Curie Actions and ERC (European Union), ANR, Labex P2IO and OCEVU, and Région Auvergne-Rhône-Alpes (France), Key Research Program of Frontier Sciences of CAS, CAS PIFI, and the Thousand Talents Program (China), RFBR, RSF and Yandex LLC (Russia), GVA, XuntaGal and GENCAT (Spain), Herchel Smith Fund, the Royal Society, the English-Speaking Union and the Leverhulme Trust (United Kingdom).

\section{A Results for other models}

Two additional boson production models are tested and the resulting upper limits are shown in figure 5. The first model is a vector boson, $A^{\prime}$, produced via Drell-Yan $q \bar{q} \rightarrow A^{\prime}$ 
and decaying to a pair of muons. The Drell-Yan production kinematics are taken from Pythia 8 [33]. These results can be interpreted as limits on dark photons since their production mode is expected to be dominated by Drell-Yan in this region of masses.

In the second model the signal is assumed to come from the decay of the $125 \mathrm{GeV}$ Higgs to two spin- $0 \phi$ bosons. Only one of the two $\phi$ bosons is required to decay to a dimuon final state, so the limit is set on $\left(\sigma_{H} / \sigma_{\mathrm{SM}}\right) \times \mathcal{B}(H \rightarrow \phi \phi) \times \mathcal{B}\left(\phi \rightarrow \mu^{+} \mu^{-}\right)$, where $\sigma_{H}$ is the $125 \mathrm{GeV}$ Higgs cross-section and $\sigma_{\mathrm{SM}}$ is its value as computed in the SM. The combination of 7 and $8 \mathrm{TeV}$ results is obtained by taking for $\sigma_{\mathrm{SM}}$ the SM gluon-gluon fusion crosssections for a $125 \mathrm{GeV}$ Higgs from ref. [55] and assuming that $\sigma_{H} / \sigma_{\mathrm{SM}}$ is independent on the centre-of-mass energy $\sqrt{s}$.

The most significant excess is $2.9 \sigma$ at $m(\phi) \simeq 12.92 \mathrm{GeV}$ in the $H \rightarrow \phi \phi$ production model hypothesis and has a $p$-value of $14 \%$, after accounting for the trials factor due to the large mass range tested in comparison to the dimuon mass resolution.

Open Access. This article is distributed under the terms of the Creative Commons Attribution License (CC-BY 4.0), which permits any use, distribution and reproduction in any medium, provided the original author(s) and source are credited.

\section{References}

[1] CMS collaboration, Observation of a new boson at a mass of $125 \mathrm{GeV}$ with the CMS experiment at the LHC, Phys. Lett. B 716 (2012) 30 [arXiv:1207.7235] [INSPIRE].

[2] ATLAS collaboration, Observation of a new particle in the search for the standard model Higgs boson with the ATLAS detector at the LHC, Phys. Lett. B 716 (2012) 1 [arXiv:1207.7214] [INSPIRE].

[3] D. Curtin et al., Exotic decays of the 125 GeV Higgs boson, Phys. Rev. D 90 (2014) 075004 [arXiv: 1312.4992] [INSPIRE].

[4] P. Fayet and S. Ferrara, Supersymmetry, Phys. Rept. 32 (1977) 249 [INSPIRE].

[5] J.R. Ellis et al., Higgs bosons in a nonminimal supersymmetric model, Phys. Rev. D 39 (1989) 844 [INSPIRE].

[6] U. Ellwanger, C. Hugonie and A.M. Teixeira, The next-to-minimal supersymmetric standard model, Phys. Rept. 496 (2010) 1 [arXiv:0910.1785] [InSPIRE].

[7] N. Arkani-Hamed, A.G. Cohen and H. Georgi, Electroweak symmetry breaking from dimensional deconstruction, Phys. Lett. B 513 (2001) 232 [hep-ph/0105239] [INSPIRE].

[8] N. Arkani-Hamed et al., The minimal moose for a little Higgs, JHEP 08 (2002) 021 [hep-ph/0206020] [INSPIRE].

[9] M. Perelstein, Little Higgs models and their phenomenology, Prog. Part. Nucl. Phys. 58 (2007) 247 [hep-ph/0512128] [inSPIRE].

[10] V. Silveira and A. Zee, Scalar phantoms, Phys. Lett. B 161 (1985) 136.

[11] J. McDonald, Gauge singlet scalars as cold dark matter, Phys. Rev. D 50 (1994) 3637 [hep-ph/0702143] [INSPIRE].

[12] C.P. Burgess, M. Pospelov and T. ter Veldhuis, The minimal model of nonbaryonic dark matter: a singlet scalar, Nucl. Phys. B 619 (2001) 709 [hep-ph/0011335] [INSPIRE]. 
[13] F. Bezrukov and D. Gorbunov, Light inflaton hunter's guide, JHEP 05 (2010) 010 [arXiv:0912.0390] [INSPIRE].

[14] F. Bezrukov and D. Gorbunov, Light inflaton after LHC8 and WMAP9 results, JHEP 07 (2013) 140 [arXiv:1303.4395] [inSPIRE].

[15] U. Haisch, J.F. Kamenik, A. Malinauskas and M. Spira, Collider constraints on light pseudoscalars, JHEP 03 (2018) 178 [arXiv:1802.02156] [INSPIRE].

[16] CMS collaboration, Search for new resonances in the diphoton final state in the mass range between 70 and $110 \mathrm{GeV}$ in pp collisions at $\sqrt{s}=8$ and $13 \mathrm{TeV}$, CMS-PAS-HIG-17-013 (2017).

[17] CMS collaboration, Search for additional neutral MSSM Higgs bosons in the $\tau \tau$ final state in proton-proton collisions at $\sqrt{s}=13 \mathrm{TeV}$, JHEP 09 (2018) 007 [arXiv: 1803. 06553] [INSPIRE].

[18] CMS collaboration, Search for a low-mass pseudoscalar higgs boson produced in association with a b $\bar{b}$ pair in pp collisions at $\sqrt{s}=8 \mathrm{TeV}$, Phys. Lett. B 758 (2016) 296 [arXiv: 1511.03610] [INSPIRE].

[19] CMS collaboration, Search for a light pseudoscalar Higgs boson in the dimuon decay channel in pp collisions at $\sqrt{s}=7$ TeV, Phys. Rev. Lett. 109 (2012) 121801 [arXiv:1206.6326] [INSPIRE].

[20] BABAR collaboration, J.P. Lees et al., Search for di-muon decays of a low-mass Higgs boson in radiative decays of the $\Upsilon(1 S)$, Phys. Rev. D 87 (2013) 031102 [Erratum ibid. D 87 (2013) 059903] [arXiv: 1210.0287] [INSPIRE].

[21] BABAR collaboration, J.P. Lees et al., Search for a low-mass scalar Higgs boson decaying to a tau pair in single-photon decays of $\Upsilon(1 S)$, Phys. Rev. D 88 (2013) 071102 [arXiv:1210.5669] [INSPIRE].

[22] LHCb collaboration, Search for hidden-sector bosons in $B^{0} \rightarrow K^{* 0} \mu^{+} \mu^{-}$decays, Phys. Rev. Lett. 115 (2015) 161802 [arXiv: 1508.04094] [INSPIRE].

[23] LHCb collaboration, Search for long-lived scalar particles in $B^{+} \rightarrow K^{+} \chi\left(\mu^{+} \mu^{-}\right)$decays, Phys. Rev. D 95 (2017) 071101 [arXiv:1612.07818] [INSPIRE].

[24] U. Haisch and J.F. Kamenik, Searching for new spin-0 resonances at LHCb, Phys. Rev. D 93 (2016) 055047 [arXiv: 1601.05110] [INSPIRE].

[25] LHCb collaboration, Search for dark photons produced in 13 TeV pp collisions, Phys. Rev. Lett. 120 (2018) 061801 [arXiv: 1710.02867] [INSPIRE].

[26] LHCb collaboration, The LHCb detector at the LHC, 2008 JINST 3 S08005 [inSPIRE].

[27] LHCb collaboration, LHCb detector performance, Int. J. Mod. Phys. A 30 (2015) 1530022 [arXiv: 1412.6352] [INSPIRE].

[28] R. Aaij et al., Performance of the LHCb Vertex Locator, 2014 JINST 9 P09007 [arXiv: 1405.7808] [INSPIRE].

[29] R. Arink et al., Performance of the LHCb Outer Tracker, 2014 JINST 9 P01002 [arXiv: 1311.3893] [INSPIRE].

[30] M. Adinolfi et al., Performance of the LHCb RICH detector at the LHC, Eur. Phys. J. C 73 (2013) 2431 [arXiv: 1211.6759] [inSPIRE]. 
[31] A.A. Alves, Jr. et al., Performance of the LHCb muon system, 2013 JINST 8 P02022 [arXiv: 1211.1346] [INSPIRE].

[32] R. Aaij et al., The LHCb trigger and its performance in 2011, 2013 JINST 8 P04022 [arXiv:1211.3055] [INSPIRE].

[33] T. Sjöstrand, S. Mrenna and P.Z. Skands, A brief introduction to PYTHIA 8.1, Comput. Phys. Commun. 178 (2008) 852 [arXiv:0710.3820] [INSPIRE].

[34] T. Sjöstrand, S. Mrenna and P.Z. Skands, PYTHIA 6.4 physics and manual, JHEP 05 (2006) 026 [hep-ph/0603175] [INSPIRE].

[35] I. Belyaev et al., Handling of the generation of primary events in Gauss, the LHCb simulation framework, J. Phys. Conf. Ser. 331 (2011) 032047 [InSPIRE].

[36] D.J. Lange, The EvtGen particle decay simulation package, Nucl. Instrum. Meth. A 462 (2001) 152 [INSPIRE].

[37] P. Golonka and Z. Was, PHOTOS Monte Carlo: a precision tool for QED corrections in Z and $W$ decays, Eur. Phys. J. C 45 (2006) 97 [hep-ph/0506026] [INSPIRE].

[38] GEANT4 collaboration, J. Allison et al., GEANT4 developments and applications, IEEE Trans. Nucl. Sci. 53 (2006) 270.

[39] GEANT4 collaboration, S. Agostinelli et al., GEANT4: a simulation toolkit, Nucl. Instrum. Meth. A 506 (2003) 250 [inSPIRE].

[40] M. Clemencic et al., The LHCb simulation application, Gauss: Design, evolution and experience, J. Phys. Conf. Ser. 331 (2011) 032023 [INSPIRE].

[41] J. Stevens and M. Williams, uBoost: a boosting method for producing uniform selection efficiencies from multivariate classifiers, 2013 JINST 8 P12013 [arXiv:1305.7248] [INSPIRE].

[42] LHCb collaboration, Measurement of the $B_{s}^{0} \rightarrow \mu^{+} \mu^{-}$branching fraction and search for $B^{0} \rightarrow \mu^{+} \mu^{-}$decays at the LHCb experiment, Phys. Rev. Lett. 111 (2013) 101805 [arXiv: 1307.5024] [INSPIRE].

[43] A. Rogozhnikov et al., Machine learning for high energy physics, Zenodo (2018).

[44] M. Pivk and F.R. Le Diberder, SPlot: a statistical tool to unfold data distributions, Nucl. Instrum. Meth. A 555 (2005) 356 [physics/0402083] [INSPIRE].

[45] G. Punzi, Sensitivity of searches for new signals and its optimization, eConf C 030908 (2003) MODT002 [physics/0308063] [INSPIRE].

[46] LHCb collaboration, Measurement of the track reconstruction efficiency at LHCb, 2015 JINST 10 P02007 [arXiv:1408.1251] [INSPIRE].

[47] L. Anderlini et al., The PIDCalib package, LHCb-PUB-2016-021 (2016).

[48] D. Martínez Santos et al., Ipanema- $\beta$ : tools and examples for HEP analysis on GPU, arXiv: 1706.01420 [INSPIRE].

[49] F. James and M. Roos, Minuit: a system for function minimization and analysis of the parameter errors and correlations, Comput. Phys. Commun. 10 (1975) 343 [INSPIRE].

[50] D. Martínez Santos and F. Dupertuis, Mass distributions marginalized over per-event errors, Nucl. Instrum. Meth. A 764 (2014) 150 [arXiv:1312.5000] [INSPIRE]. 
[51] T. Skwarnicki, A study of the radiative cascade transitions between the $\Upsilon^{\prime}$ and $\Upsilon$ resonances, Ph.D. thesis, Institute of Nuclear Physics, Krakow Poland (1986) [DESY-F31-86-02].

[52] T. Junk, Confidence level computation for combining searches with small statistics, Nucl. Instrum. Meth. A 434 (1999) 435 [hep-ex/9902006] [INSPIRE].

[53] A.L. Read, Presentation of search results: the CL(s) technique, J. Phys. G 28 (2002) 2693 [INSPIRE].

[54] LHCb collaboration, Precision luminosity measurements at LHCb, 2014 JINST 9 P12005 [arXiv: 1410.0149] [INSPIRE].

[55] LHC Higgs Cross Section Working Group, S. Heinemeyer et al., Handbook of LHC Higgs Cross Sections: 3. Higgs properties: report of the LHC Higgs Cross Section Working Group, CERN-2013-004 (2013). 


\section{The LHCb collaboration}

R. Aaij ${ }^{43}$, B. Adeva ${ }^{39}$, M. Adinolfi ${ }^{48}$, Z. Ajaltouni ${ }^{5}$, S. Akar ${ }^{59}$, P. Albicocco ${ }^{19}$, J. Albrecht ${ }^{10}$, F. Alessio ${ }^{40}$, M. Alexander ${ }^{53}$, A. Alfonso Albero ${ }^{38}$, S. Ali ${ }^{43}$, G. Alkhazov ${ }^{31}$, P. Alvarez Cartelle ${ }^{55}$, A.A. Alves $\mathrm{Jr}^{59}$, S. Amato ${ }^{2}$, S. Amerio ${ }^{23}$, Y. Amhis ${ }^{7}$, L. An ${ }^{3}$, L. Anderlini ${ }^{18}$, G. Andreassi ${ }^{41}$, M. Andreotti ${ }^{17, g}$, J.E. Andrews ${ }^{60}$, R.B. Appleby ${ }^{56}$, F. Archilli ${ }^{43}$, P. d'Argent ${ }^{12}$, J. Arnau Romeu ${ }^{6}$, A. Artamonov ${ }^{37}$, M. Artuso ${ }^{61}$, E. Aslanides ${ }^{6}$, M. Atzeni ${ }^{42}$, G. Auriemma ${ }^{26}$, S. Bachmann ${ }^{12}$, J.J. Back ${ }^{50}$, S. Baker ${ }^{55}$, V. Balagura ${ }^{7, b}$, W. Baldini ${ }^{17}$, A. Baranov ${ }^{35}$, R.J. Barlow ${ }^{56}$, S. Barsuk ${ }^{7}$, W. Barter ${ }^{56}$, F. Baryshnikov ${ }^{32}$, V. Batozskaya ${ }^{29}$, V. Battista ${ }^{41}$, A. Bay ${ }^{41}$, J. Beddow ${ }^{53}$, F. Bedeschi ${ }^{24}$, I. Bediaga ${ }^{1}$, A. Beiter ${ }^{61}$, L.J. Bel ${ }^{43}$, N. Beliy ${ }^{63}$, V. Bellee ${ }^{41}$, N. Belloli ${ }^{21, i}$, K. Belous ${ }^{37}$, I. Belyaev ${ }^{32,40}$, E. Ben-Haim ${ }^{8}$, G. Bencivenni ${ }^{19}$, S. Benson ${ }^{43}$, S. Beranek ${ }^{9}$, A. Berezhnoy ${ }^{33}$, R. Bernet ${ }^{42}$, D. Berninghoff ${ }^{12}$, E. Bertholet ${ }^{8}$, A. Bertolin $^{23}$, C. Betancourt ${ }^{42}$, F. Betti ${ }^{15,40}$, M.O. Bettler ${ }^{49}$, M. van Beuzekom ${ }^{43}$, Ia. Bezshyiko ${ }^{42}$, S. Bifani ${ }^{47}$, P. Billoir ${ }^{8}$, A. Birnkraut ${ }^{10}$, A. Bizzeti ${ }^{18, u}$, M. Bjørn ${ }^{57}$, T. Blake ${ }^{50}$, F. Blanc ${ }^{41}$, S. Blusk ${ }^{61}$, V. Bocci ${ }^{26}$, O. Boente Garcia ${ }^{39}$, T. Boettcher ${ }^{58}$, A. Bondar ${ }^{36, w}$, N. Bondar ${ }^{31}$, S. Borghi ${ }^{56,40}$, M. Borisyak ${ }^{35}$, M. Borsato ${ }^{39,40}$, F. Bossu ${ }^{7}$, M. Boubdir ${ }^{9}$, T.J.V. Bowcock ${ }^{54}$, E. Bowen ${ }^{42}$, C. Bozzi ${ }^{17,40}$, S. Braun ${ }^{12}$, M. Brodski ${ }^{40}$, J. Brodzicka ${ }^{27}$, D. Brundu ${ }^{16}$, E. Buchanan ${ }^{48}$, C. Burr ${ }^{56}$, A. Bursche ${ }^{16}$, J. Buytaert ${ }^{40}$, W. Byczynski ${ }^{40}$, S. Cadeddu ${ }^{16}$, H. Cai ${ }^{64}$, R. Calabrese $^{17, g}$, R. Calladine ${ }^{47}$, M. Calvi ${ }^{21, i}$, M. Calvo Gomez ${ }^{38, m}$, A. Camboni ${ }^{38, m}$, P. Campana ${ }^{19}$, D.H. Campora Perez ${ }^{40}$, L. Capriotti ${ }^{56}$, A. Carbone ${ }^{15, e}$, G. Carboni ${ }^{25}$, R. Cardinale $^{20, h}$, A. Cardini ${ }^{16}$, P. Carniti ${ }^{21, i}$, L. Carson ${ }^{52}$, K. Carvalho Akiba ${ }^{2}$, G. Casse ${ }^{54}$, L. Cassina ${ }^{21}$, M. Cattaneo ${ }^{40}$, G. Cavallero ${ }^{20, h}$, R. Cenci ${ }^{24, p}$, D. Chamont ${ }^{7}$, M.G. Chapman ${ }^{48}$, M. Charles ${ }^{8}$, Ph. Charpentier $^{40}$,

G. Chatzikonstantinidis ${ }^{47}$, M. Chefdeville ${ }^{4}$, S. Chen ${ }^{16}$, S.-G. Chitic ${ }^{40}$, V. Chobanova ${ }^{39}$, M. Chrzaszcz ${ }^{42}$, A. Chubykin ${ }^{31}$, P. Ciambrone ${ }^{19}$, X. Cid Vidal ${ }^{39}$, G. Ciezarek $^{40}$, P.E.L. Clarke ${ }^{52}$, M. Clemencic ${ }^{40}$, H.V. Cliff ${ }^{49}$, J. Closier ${ }^{40}$, V. Coco ${ }^{40}$, J. $\operatorname{Cogan}^{6}$, E. $\operatorname{Cogneras}^{5}$, V. Cogoni ${ }^{16, f}$, L. Cojocariu ${ }^{30}$, P. Collins ${ }^{40}$, T. Colombo ${ }^{40}$, A. Comerma-Montells ${ }^{12}$, A. Contu ${ }^{16}$, G. Coombs ${ }^{40}$,

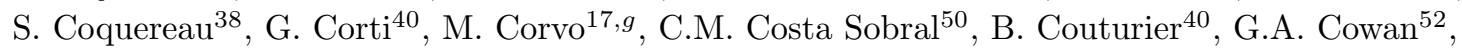
D.C. Craik ${ }^{58}$, A. Crocombe ${ }^{50}$, M. Cruz Torres ${ }^{1}$, R. Currie ${ }^{52}$, C. D'Ambrosio ${ }^{40}$,

F. Da Cunha Marinho ${ }^{2}$, C.L. Da Silva ${ }^{73}$, E. Dall'Occo ${ }^{43}$, J. Dalseno ${ }^{48}$, A. Danilina ${ }^{32}$, A. Davis ${ }^{3}$, O. De Aguiar Francisco ${ }^{40}$, K. De Bruyn ${ }^{40}$, S. De Capua ${ }^{56}$, M. De Cian ${ }^{41}$, J.M. De Miranda ${ }^{1}$, L. De Paula ${ }^{2}$, M. De Serio ${ }^{14, d}$, P. De Simone ${ }^{19}$, C.T. Dean ${ }^{53}$, D. Decamp ${ }^{4}$, L. Del Buono ${ }^{8}$, B. Delaney ${ }^{49}$, H.-P. Dembinski ${ }^{11}$, M. Demmer ${ }^{10}$, A. Dendek ${ }^{28}$, D. Derkach ${ }^{35}$, O. Deschamps ${ }^{5}$, F. Dettori ${ }^{54}$, B. Dey ${ }^{65}$, A. Di Canto ${ }^{40}$, P. Di Nezza ${ }^{19}$, S. Didenko ${ }^{69}$, H. Dijkstra ${ }^{40}$, F. Dordei ${ }^{40}$, M. Dorigo ${ }^{40}$, A. Dosil Suárez ${ }^{39}$, L. Douglas ${ }^{53}$, A. Dovbnya ${ }^{45}$, K. Dreimanis ${ }^{54}$, L. Dufour ${ }^{43}$, G. Dujany ${ }^{8}$, P. Durante ${ }^{40}$, J.M. Durham ${ }^{73}$, D. Dutta ${ }^{56}$, R. Dzhelyadin ${ }^{37}$, M. Dziewiecki ${ }^{12}$,

A. Dziurda ${ }^{40}$, A. Dzyuba ${ }^{31}$, S. Easo ${ }^{51}$, U. Egede ${ }^{55}$, V. Egorychev ${ }^{32}$, S. Eidelman ${ }^{36, w}$, S. Eisenhardt ${ }^{52}$, U. Eitschberger ${ }^{10}$, R. Ekelhof ${ }^{10}$, L. Eklund ${ }^{53}$, S. Ely ${ }^{61}$, A. Ene ${ }^{30}$, S. Escher ${ }^{9}$, S. Esen ${ }^{12}$, H.M. Evans ${ }^{49}$, T. Evans ${ }^{57}$, A. Falabella ${ }^{15}$, N. Farley ${ }^{47}$, S. Farry ${ }^{54}$, D. Fazzini ${ }^{21,40, i}$, L. Federici ${ }^{25}$, G. Fernandez ${ }^{38}$, P. Fernandez Declara ${ }^{40}$, A. Fernandez Prieto ${ }^{39}$, F. Ferrari ${ }^{15}$, L. Ferreira Lopes ${ }^{41}$, F. Ferreira Rodrigues ${ }^{2}$, M. Ferro-Luzzi ${ }^{40}$, S. Filippov ${ }^{34}$, R.A. Fini ${ }^{14}$, M. Fiorini ${ }^{17, g}$, M. Firlej ${ }^{28}$, C. Fitzpatrick ${ }^{41}$, T. Fiutowski ${ }^{28}$, F. Fleuret ${ }^{7, b}$, M. Fontana ${ }^{16,40}$, F. Fontanelli ${ }^{20, h}$, R. Forty ${ }^{40}$, V. Franco Lima ${ }^{54}$, M. Frank ${ }^{40}$, C. Frei ${ }^{40}$, J. Fu ${ }^{22, q}$, W. Funk ${ }^{40}$, C. Färber ${ }^{40}$, E. Gabriel ${ }^{52}$, A. Gallas Torreira ${ }^{39}$, D. Galli15,e, S. Gallorini ${ }^{23}$, S. Gambetta ${ }^{52}$,

M. Gandelman ${ }^{2}$, P. Gandini ${ }^{22}$, Y. Gao ${ }^{3}$, L.M. Garcia Martin ${ }^{71}$, B. Garcia Plana ${ }^{39}$,

J. García Pardiñas ${ }^{39}$, J. Garra Tico ${ }^{49}$, L. Garrido ${ }^{38}$, D. Gascon ${ }^{38}$, C. Gaspar ${ }^{40}$, L. Gavardi ${ }^{10}$,

G. Gazzoni ${ }^{5}$, D. Gerick ${ }^{12}$, E. Gersabeck ${ }^{56}$, M. Gersabeck ${ }^{56}$, T. Gershon ${ }^{50}$, Ph. Ghez ${ }^{4}$, S. Giani ${ }^{41}$,

V. Gibson ${ }^{49}$, O.G. Girard ${ }^{41}$, L. Giubega ${ }^{30}$, K. Gizdov ${ }^{52}$, V.V. Gligorov ${ }^{8}$, D. Golubkov ${ }^{32}$,

A. Golutvin ${ }^{55,69}$, A. Gomes ${ }^{1, a}$, I.V. Gorelov ${ }^{33}$, C. Gotti ${ }^{21, i}$, E. Govorkova ${ }^{43}$, J.P. Grabowski ${ }^{12}$, 
R. Graciani Diaz ${ }^{38}$, L.A. Granado Cardoso ${ }^{40}$, E. Graugés ${ }^{38}$, E. Graverini ${ }^{42}$, G. Graziani ${ }^{18}$, A. Grecu $^{30}$, R. Greim ${ }^{43}$, P. Griffith ${ }^{16}$, L. Grillo ${ }^{56}$, L. Gruber ${ }^{40}$, B.R. Gruberg Cazon ${ }^{57}$, O. Grünberg ${ }^{67}$, E. Gushchin ${ }^{34}$, Yu. Guz ${ }^{37,40}$, T. Gys ${ }^{40}$, C. Göbel ${ }^{62}$, T. Hadavizadeh ${ }^{57}$, C. Hadjivasiliou ${ }^{5}$, G. Haefeli ${ }^{41}$, C. Haen ${ }^{40}$, S.C. Haines ${ }^{49}$, B. Hamilton ${ }^{60}$, X. Han ${ }^{12}$, T.H. Hancock ${ }^{57}$, S. Hansmann-Menzemer ${ }^{12}$, N. Harnew ${ }^{57}$, S.T. Harnew ${ }^{48}$, C. Hasse ${ }^{40}$, M. Hatch ${ }^{40}$, J. He ${ }^{63}$, M. Hecker ${ }^{55}$, K. Heinicke ${ }^{10}$, A. Heister ${ }^{9}$, K. Hennessy ${ }^{54}$, L. Henry ${ }^{71}$, E. van Herwijnen ${ }^{40}$, M. Heß ${ }^{67}$, A. Hicheur ${ }^{2}$, D. Hill ${ }^{57}$, P.H. Hopchev ${ }^{41}$, W. Hu ${ }^{65}$, W. Huang ${ }^{63}$, Z.C. Huard ${ }^{59}$, W. Hulsbergen ${ }^{43}$, T. Humair ${ }^{55}$, M. Hushchyn ${ }^{35}$, D. Hutchcroft ${ }^{54}$, P. Ibis $^{10}$, M. Idzik $^{28}$, P. Ilten ${ }^{47}$, K. Ivshin ${ }^{31}$, R. Jacobsson ${ }^{40}$, J. Jalocha ${ }^{57}$, E. Jans ${ }^{43}$, A. Jawahery ${ }^{60}$, F. Jiang ${ }^{3}$, M. John ${ }^{57}$, D. Johnson ${ }^{40}$, C.R. Jones ${ }^{49}$, C. Joram ${ }^{40}$, B. Jost ${ }^{40}$, N. Jurik ${ }^{57}$, S. Kandybei ${ }^{45}$, M. Karacson ${ }^{40}$, J.M. Kariuki ${ }^{48}$, S. Karodia ${ }^{53}$, N. Kazeev ${ }^{35}$, M. Kecke ${ }^{12}$, F. Keizer ${ }^{49}$, M. Kelsey ${ }^{61}$, M. Kenzie ${ }^{49}$, T. Ketel ${ }^{44}$, E. Khairullin ${ }^{35}$, B. Khanji ${ }^{12}$, C. Khurewathanakul ${ }^{41}$, K.E. Kim ${ }^{61}$, T. Kirn ${ }^{9}$, S. Klaver ${ }^{19}$, K. Klimaszewski ${ }^{29}$, T. Klimkovich ${ }^{11}$, S. Koliiev ${ }^{46}$, M. Kolpin ${ }^{12}$, R. Kopecna ${ }^{12}$, P. Koppenburg ${ }^{43}$, S. Kotriakhova ${ }^{31}$, M. Kozeiha ${ }^{5}$, L. Kravchuk ${ }^{34}$, M. Kreps ${ }^{50}$, F. Kress ${ }^{55}$, P. Krokovny ${ }^{36, w}$, W. Krupa ${ }^{28}$, W. Krzemien ${ }^{29}$, W. Kucewicz ${ }^{27, l}$, M. Kucharczyk ${ }^{27}$, V. Kudryavtsev ${ }^{36, w}$, A.K. Kuonen ${ }^{41}$, T. Kvaratskheliya ${ }^{32,40}$,

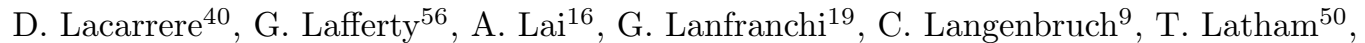
C. Lazzeroni ${ }^{47}$, R. Le $\mathrm{Gac}^{6}$, A. Leflat ${ }^{33,40}$, J. Lefrançois ${ }^{7}$, R. Lefèvre ${ }^{5}$, F. Lemaitre ${ }^{40}$, E. Lemos Cid $^{39}$, P. Lenisa ${ }^{17}$, O. Leroy ${ }^{6}$, T. Lesiak ${ }^{27}$, B. Leverington ${ }^{12}$, P.-R. Li ${ }^{63}$, T. Li $^{3}$, Z. Li $^{61}$, X. Liang ${ }^{61}$, T. Likhomanenko ${ }^{68}$, R. Lindner ${ }^{40}$, F. Lionetto ${ }^{42}$, V. Lisovskyi ${ }^{7}$, X. Liu ${ }^{3}$, D. Loh ${ }^{50}$, A. Loi ${ }^{16}$, I. Longstaff ${ }^{53}$, J.H. Lopes ${ }^{2}$, D. Lucchesi ${ }^{23, o}$, M. Lucio Martinez ${ }^{39}$, A. Lupato ${ }^{23}$,

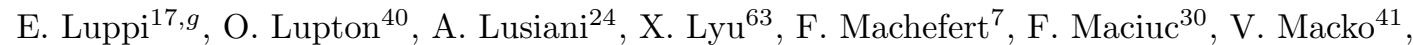
P. Mackowiak ${ }^{10}$, S. Maddrell-Mander ${ }^{48}$, O. Maev $^{31,40}$, K. Maguire $^{56}$, D. Maisuzenko ${ }^{31}$, M.W. Majewski ${ }^{28}$, S. Malde ${ }^{57}$, B. Malecki ${ }^{27}$, A. Malinin ${ }^{68}$, T. Maltsev ${ }^{36, w}$, G. Manca ${ }^{16, f}$, G. Mancinelli ${ }^{6}$, D. Marangotto ${ }^{22, q}$, J. Maratas ${ }^{5, v}$, J.F. Marchand ${ }^{4}$, U. Marconi ${ }^{15}$, C. Marin Benito ${ }^{38}$, M. Marinangeli ${ }^{41}$, P. Marino ${ }^{41}$, J. Marks ${ }^{12}$, G. Martellotti ${ }^{26}$, M. Martin ${ }^{6}$, M. Martinelli ${ }^{41}$, D. Martinez Santos ${ }^{39}$, F. Martinez Vidal ${ }^{71}$, A. Massafferri ${ }^{1}$, R. Matev ${ }^{40}$, A. Mathad ${ }^{50}$, Z. Mathe ${ }^{40}$, C. Matteuzzi ${ }^{21}$, A. Mauri ${ }^{42}$, E. Maurice ${ }^{7, b}$, B. Maurin ${ }^{41}$, A. Mazurov ${ }^{47}$, M. McCann ${ }^{55,40}$, A. McNab ${ }^{56}$, R. McNulty ${ }^{13}$, J.V. Mead ${ }^{54}$, B. Meadows ${ }^{59}$, C. Meaux ${ }^{6}$, F. Meier ${ }^{10}$, N. Meinert ${ }^{67}$, D. Melnychuk ${ }^{29}$, M. Merk ${ }^{43}$, A. Merli $^{22, q}$, E. Michielin ${ }^{23}$, D.A. Milanes ${ }^{66}$, E. Millard ${ }^{50}$, M.-N. Minard ${ }^{4}$, L. Minzoni ${ }^{17}$, D.S. Mitzel ${ }^{12}$, A. Mogini $^{8}$, J. Molina Rodriguez ${ }^{1}$, T. Mombächer ${ }^{10}$, I.A. Monroy $^{66}$, S. Monteil ${ }^{5}$, M. Morandin ${ }^{23}$, G. Morello ${ }^{19}$, M.J. Morello ${ }^{24, t}$, O. Morgunova ${ }^{68}$, J. Moron ${ }^{28}$, A.B. Morris ${ }^{52}$, R. Mountain ${ }^{61}$, F. Muheim ${ }^{52}$, M. Mulder ${ }^{43}$, D. Müller ${ }^{40}$, J. Müller ${ }^{10}$, K. Müller ${ }^{42}$, V. Müller ${ }^{10}$, P. Naik ${ }^{48}$, T. Nakada ${ }^{41}$, R. Nandakumar ${ }^{51}$, A. Nandi ${ }^{57}$, I. Nasteva ${ }^{2}$, M. Needham ${ }^{52}$, N. Neri ${ }^{22}$, S. Neubert ${ }^{12}$, N. Neufeld ${ }^{40}$, M. Neuner ${ }^{12}$, T.D. Nguyen ${ }^{41}$, C. Nguyen-Mau ${ }^{41, n}$, S. Nieswand ${ }^{9}$, R. Niet ${ }^{10}$, N. Nikitin ${ }^{33}$, A. Nogay ${ }^{68}$, D.P. O'Hanlon ${ }^{15}$, A. Oblakowska-Mucha ${ }^{28}$, V. Obraztsov ${ }^{37}$, S. Ogilvy ${ }^{19}$, R. Oldeman ${ }^{16, f}$, C.J.G. Onderwater ${ }^{72}$, A. Ossowska ${ }^{27}$, J.M. Otalora Goicochea ${ }^{2}$, P. Owen ${ }^{42}$, A. Oyanguren ${ }^{71}$, P.R. Pais ${ }^{41}$, A. Palano ${ }^{14}$, M. Palutan ${ }^{19,40}$, G. Panshin ${ }^{70}$, A. Papanestis ${ }^{51}$, M. Pappagallo ${ }^{52}$, L.L. Pappalardo ${ }^{17, g}$, W. Parker ${ }^{60}$, C. Parkes $^{56}$, G. Passaleva ${ }^{18,40}$, A. Pastore ${ }^{14}$, M. Patel ${ }^{55}$, C. Patrignani ${ }^{15, e}$, A. Pearce ${ }^{40}$, A. Pellegrino ${ }^{43}$, G. Penso ${ }^{26}$, M. Pepe Altarelli ${ }^{40}$, S. Perazzini ${ }^{40}$, D. Pereima ${ }^{32}$, P. Perret ${ }^{5}$, L. Pescatore ${ }^{41}$, K. Petridis ${ }^{48}$, A. Petrolini ${ }^{20, h}$,

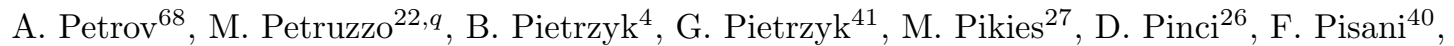
A. Pistone ${ }^{20, h}$, A. Piucci ${ }^{12}$, V. Placinta ${ }^{30}$, S. Playfer ${ }^{52}$, M. Plo Casasus ${ }^{39}$, F. Polci ${ }^{8}$, M. Poli Lener ${ }^{19}$, A. Poluektov ${ }^{50}$, N. Polukhina ${ }^{69}$, I. Polyakov ${ }^{61}$, E. Polycarpo ${ }^{2}$, G.J. Pomery ${ }^{48}$, S. Ponce ${ }^{40}$, A. Popov ${ }^{37}$, D. Popov ${ }^{11,40}$, S. Poslavskiii ${ }^{37}$, C. Potterat ${ }^{2}$, E. Price ${ }^{48}$, J. Prisciandaro ${ }^{39}$, C. Prouve ${ }^{48}$, V. Pugatch ${ }^{46}$, A. Puig Navarro ${ }^{42}$, H. Pullen ${ }^{57}$, G. Punzi ${ }^{24, p}$, W. Qian ${ }^{50}$, J. Qin ${ }^{63}$, R. Quagliani ${ }^{8}$, B. Quintana ${ }^{5}$, B. Rachwal ${ }^{28}$, J.H. Rademacker ${ }^{48}$, M. Rama ${ }^{24}$, M. Ramos Pernas ${ }^{39}$, 
M.S. Rangel ${ }^{2}$, F. Ratnikov ${ }^{35, x}$, G. Raven ${ }^{44}$, M. Ravonel Salzgeber ${ }^{40}$, M. Reboud $^{4}$, F. Redi $^{41}$, S. Reichert ${ }^{10}$, A.C. dos Reis ${ }^{1}$, C. Remon Alepuz ${ }^{71}$, V. Renaudin ${ }^{7}$, S. Ricciardi ${ }^{51}$, S. Richards ${ }^{48}$, K. Rinnert ${ }^{54}$, P. Robbe ${ }^{7}$, A. Robert ${ }^{8}$, A.B. Rodrigues ${ }^{41}$, E. Rodrigues ${ }^{59}$, J.A. Rodriguez Lopez ${ }^{66}$, A. Rogozhnikov ${ }^{35}$, S. Roiser ${ }^{40}$, A. Rollings ${ }^{57}$, V. Romanovskiy ${ }^{37}$, A. Romero Vidal ${ }^{39,40}$, M. Rotondo ${ }^{19}$, M.S. Rudolph ${ }^{61}$, T. Ruf ${ }^{40}$, J. Ruiz Vidal ${ }^{71}$, J.J. Saborido Silva ${ }^{39}$, N. Sagidova ${ }^{31}$, B. Saitta ${ }^{16, f}$, V. Salustino Guimaraes ${ }^{62}$, C. Sanchez Mayordomo ${ }^{71}$, B. Sanmartin Sedes ${ }^{39}$, R. Santacesaria ${ }^{26}$, C. Santamarina Rios ${ }^{39}$, M. Santimaria ${ }^{19}$, E. Santovetti ${ }^{25, j}$, G. Sarpis ${ }^{56}$, A. Sarti ${ }^{19, k}$, C. Satriano ${ }^{26, s}$, A. Satta ${ }^{25}$, D. Savrina ${ }^{32,33}$, S. Schael ${ }^{9}$, M. Schellenberg ${ }^{10}$, M. Schiller ${ }^{53}$, H. Schindler ${ }^{40}$, M. Schmelling ${ }^{11}$, T. Schmelzer ${ }^{10}$, B. Schmidt ${ }^{40}$, O. Schneider ${ }^{41}$, A. Schopper ${ }^{40}$, H.F. Schreiner ${ }^{59}$, M. Schubiger ${ }^{41}$, M.H. Schune ${ }^{7,40}$, R. Schwemmer ${ }^{40}$, B. Sciascia ${ }^{19}$, A. Sciubba ${ }^{26, k}$, A. Semennikov ${ }^{32}$, E.S. Sepulveda ${ }^{8}$, A. Sergi ${ }^{47,40}$, N. Serra ${ }^{42}$, J. Serrano ${ }^{6}$, L. Sestini ${ }^{23}$, P. Seyfert ${ }^{40}$, M. Shapkin ${ }^{37}$, Y. Shcheglov ${ }^{31, \dagger}$, T. Shears ${ }^{54}$, L. Shekhtman ${ }^{36, w}$, V. Shevchenko ${ }^{68}$, B.G. Siddi ${ }^{17}$, R. Silva Coutinho ${ }^{42}$, L. Silva de Oliveira ${ }^{2}$, G. Simi ${ }^{23, o}$, S. Simone ${ }^{14, d}$, N. Skidmore ${ }^{48}$, T. Skwarnicki ${ }^{61}$, I.T. Smith ${ }^{52}$, M. Smith ${ }^{55}$, l. Soares Lavra ${ }^{1}$, M.D. Sokoloff ${ }^{59}$, F.J.P. Soler ${ }^{53}$, B. Souza De Paula ${ }^{2}$, B. Spaan ${ }^{10}$, P. Spradlin ${ }^{53}$, F. Stagni ${ }^{40}$, M. Stahl ${ }^{12}$, S. Stahl ${ }^{40}$, P. Stefko ${ }^{41}$, S. Stefkova ${ }^{55}$, O. Steinkamp ${ }^{42}$, S. Stemmle ${ }^{12}$, O. Stenyakin ${ }^{37}$, M. Stepanova ${ }^{31}$, H. Stevens ${ }^{10}$, S. Stone ${ }^{61}$, B. Storaci ${ }^{42}$, S. Stracka ${ }^{24, p}$, M.E. Stramaglia ${ }^{41}$, M. Straticiuc ${ }^{30}$, U. Straumann ${ }^{42}$, S. Strokov ${ }^{70}$, J. Sun ${ }^{3}$, L. Sun ${ }^{64}$, K. Swientek ${ }^{28}$, V. Syropoulos ${ }^{44}$, T. Szumlak ${ }^{28}$, M. Szymanski ${ }^{63}$, S. T'Jampens ${ }^{4}$, A. Tayduganov ${ }^{6}$, T. Tekampe ${ }^{10}$, G. Tellarini ${ }^{17}$, F. Teubert ${ }^{40}$, E. Thomas ${ }^{40}$, J. van Tilburg ${ }^{43}$, M.J. Tilley ${ }^{55}$, V. Tisserand ${ }^{5}$, M. Tobin ${ }^{41}$, S. Tolk ${ }^{40}$, L. Tomassetti ${ }^{17, g}$, D. Tonelli ${ }^{24}$, R. Tourinho Jadallah Aoude ${ }^{1}$, E. Tournefier ${ }^{4}$, M. Traill ${ }^{53}$, M.T. $\operatorname{Tran}^{41}$, M. Tresch ${ }^{42}$, A. Trisovic ${ }^{49}$, A. Tsaregorodtsev ${ }^{6}$, A. Tully ${ }^{49}$, N. Tuning ${ }^{43,40}$, A. Ukleja ${ }^{29}$, A. Usachov ${ }^{7}$, A. Ustyuzhanin ${ }^{35}$, U. Uwer ${ }^{12}$, C. Vacca ${ }^{16, f}$, A. Vagner ${ }^{70}$, V. Vagnoni ${ }^{15}$, A. Valassi ${ }^{40}$, S. Valat ${ }^{40}$, G. Valenti ${ }^{15}$, R. Vazquez Gomez ${ }^{40}$, P. Vazquez Regueiro ${ }^{39}$, S. Vecchi ${ }^{17}$, M. van Veghel ${ }^{43}$, J.J. Velthuis ${ }^{48}$, M. Veltri ${ }^{18, r}$, G. Veneziano ${ }^{57}$, A. Venkateswaran ${ }^{61}$, T.A. Verlage ${ }^{9}$, M. Vernet ${ }^{5}$, M. Vesterinen ${ }^{57}$, J.V. Viana Barbosa ${ }^{40}$, D. Vieira ${ }^{63}$, M. Vieites Diaz ${ }^{39}$, H. Viemann ${ }^{67}$, X. Vilasis-Cardona ${ }^{38, m}$, A. Vitkovskiy ${ }^{43}$, M. Vitti ${ }^{49}$, V. Volkov ${ }^{33}$, A. Vollhardt ${ }^{42}$, B. Voneki ${ }^{40}$, A. Vorobyev ${ }^{31}$, V. Vorobyev ${ }^{36, w}$, C. Voß ${ }^{9}$, J.A. de $\operatorname{Vries}^{43}$, C. Vázquez Sierra ${ }^{43}$, R. Waldi ${ }^{67}$, J. Walsh ${ }^{24}$, J. Wang ${ }^{61}$, Y. Wang ${ }^{65}$, Z. Wang ${ }^{42}$, D.R. Ward ${ }^{49}$, H.M. Wark ${ }^{54}$, N.K. Watson ${ }^{47}$, D. Websdale ${ }^{55}$, A. Weiden ${ }^{42}$, C. Weisser ${ }^{58}$, M. Whitehead ${ }^{9}$, J. Wicht ${ }^{50}$, G. Wilkinson ${ }^{57}$, M. Wilkinson ${ }^{61}$, M.R.J. Williams ${ }^{56}$, M. Williams ${ }^{58}$, T. Williams ${ }^{47}$, F.F. Wilson ${ }^{51,40}$, J. Wimberley ${ }^{60}$, M. Winn ${ }^{7}$, J. Wishahi ${ }^{10}$, W. Wislicki ${ }^{29}$,

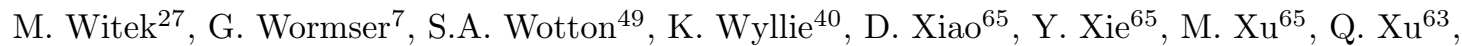

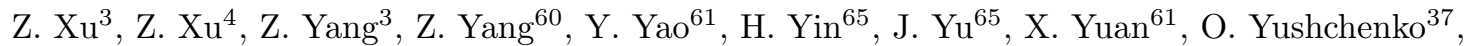
K.A. Zarebski ${ }^{47}$, M. Zavertyaev ${ }^{11, c}$, L. Zhang ${ }^{3}$, Y. Zhang ${ }^{7}$, A. Zhelezov ${ }^{12}$, Y. Zheng ${ }^{63}$, X. Zhu ${ }^{3}$, V. Zhukov ${ }^{9,33}$, J.B. Zonneveld ${ }^{52}$, S. Zucchelli ${ }^{15}$

1 Centro Brasileiro de Pesquisas Físicas (CBPF), Rio de Janeiro, Brazil

2 Universidade Federal do Rio de Janeiro (UFRJ), Rio de Janeiro, Brazil

3 Center for High Energy Physics, Tsinghua University, Beijing, China

4 Univ. Grenoble Alpes, Univ. Savoie Mont Blanc, CNRS, IN2P3-LAPP, Annecy, France

5 Clermont Université, Université Blaise Pascal, CNRS/IN2P3, LPC, Clermont-Ferrand, France

6 Aix Marseille Univ, CNRS/IN2P3, CPPM, Marseille, France

7 LAL, Univ. Paris-Sud, CNRS/IN2P3, Université Paris-Saclay, Orsay, France

8 LPNHE, Université Pierre et Marie Curie, Université Paris Diderot, CNRS/IN2P3, Paris, France

9 I. Physikalisches Institut, RWTH Aachen University, Aachen, Germany

10 Fakultät Physik, Technische Universität Dortmund, Dortmund, Germany

11 Max-Planck-Institut für Kernphysik (MPIK), Heidelberg, Germany

12 Physikalisches Institut, Ruprecht-Karls-Universität Heidelberg, Heidelberg, Germany 
13 School of Physics, University College Dublin, Dublin, Ireland

14 Sezione INFN di Bari, Bari, Italy

15 Sezione INFN di Bologna, Bologna, Italy

16 Sezione INFN di Cagliari, Cagliari, Italy

17 Universita e INFN, Ferrara, Ferrara, Italy

18 Sezione INFN di Firenze, Firenze, Italy

19 Laboratori Nazionali dell'INFN di Frascati, Frascati, Italy

20 Sezione INFN di Genova, Genova, Italy

21 Sezione INFN di Milano Bicocca, Milano, Italy

22 Sezione di Milano, Milano, Italy

23 Sezione INFN di Padova, Padova, Italy

24 Sezione INFN di Pisa, Pisa, Italy

25 Sezione INFN di Roma Tor Vergata, Roma, Italy

26 Sezione INFN di Roma La Sapienza, Roma, Italy

27 Henryk Niewodniczanski Institute of Nuclear Physics Polish Academy of Sciences, Kraków, Poland

28 AGH - University of Science and Technology, Faculty of Physics and Applied Computer Science, Kraków, Poland

29 National Center for Nuclear Research (NCBJ), Warsaw, Poland

30 Horia Hulubei National Institute of Physics and Nuclear Engineering, Bucharest-Magurele, Romania

31 Petersburg Nuclear Physics Institute (PNPI), Gatchina, Russia

32 Institute of Theoretical and Experimental Physics (ITEP), Moscow, Russia

33 Institute of Nuclear Physics, Moscow State University (SINP MSU), Moscow, Russia

34 Institute for Nuclear Research of the Russian Academy of Sciences (INR RAS), Moscow, Russia

35 Yandex School of Data Analysis, Moscow, Russia

36 Budker Institute of Nuclear Physics (SB RAS), Novosibirsk, Russia

37 Institute for High Energy Physics (IHEP), Protvino, Russia

38 ICCUB, Universitat de Barcelona, Barcelona, Spain

39 Instituto Galego de Física de Altas Enerxías (IGFAE), Universidade de Santiago de Compostela, Santiago de Compostela, Spain

40 European Organization for Nuclear Research (CERN), Geneva, Switzerland

41 Institute of Physics, Ecole Polytechnique Fédérale de Lausanne (EPFL), Lausanne, Switzerland

42 Physik-Institut, Universität Zürich, Zürich, Switzerland

43 Nikhef National Institute for Subatomic Physics, Amsterdam, The Netherlands

44 Nikhef National Institute for Subatomic Physics and VU University Amsterdam, Amsterdam, The Netherlands

45 NSC Kharkiv Institute of Physics and Technology (NSC KIPT), Kharkiv, Ukraine

46 Institute for Nuclear Research of the National Academy of Sciences (KINR), Kyiv, Ukraine

47 University of Birmingham, Birmingham, United Kingdom

48 H.H. Wills Physics Laboratory, University of Bristol, Bristol, United Kingdom

49 Cavendish Laboratory, University of Cambridge, Cambridge, United Kingdom

50 Department of Physics, University of Warwick, Coventry, United Kingdom

51 STFC Rutherford Appleton Laboratory, Didcot, United Kingdom

52 School of Physics and Astronomy, University of Edinburgh, Edinburgh, United Kingdom

53 School of Physics and Astronomy, University of Glasgow, Glasgow, United Kingdom

54 Oliver Lodge Laboratory, University of Liverpool, Liverpool, United Kingdom

55 Imperial College London, London, United Kingdom

56 School of Physics and Astronomy, University of Manchester, Manchester, United Kingdom

57 Department of Physics, University of Oxford, Oxford, United Kingdom

58 Massachusetts Institute of Technology, Cambridge, MA, United States

59 University of Cincinnati, Cincinnati, OH, United States

60 University of Maryland, College Park, MD, United States 
61 Syracuse University, Syracuse, NY, United States

62 Pontifícia Universidade Católica do Rio de Janeiro (PUC-Rio), Rio de Janeiro, Brazil, associated $t^{2}$

63 University of Chinese Academy of Sciences, Beijing, China, associated to ${ }^{3}$

64 School of Physics and Technology, Wuhan University, Wuhan, China, associated to ${ }^{3}$

65 Institute of Particle Physics, Central China Normal University, Wuhan, Hubei, China, associated ${ }^{3}$

66 Departamento de Fisica, Universidad Nacional de Colombia, Bogota, Colombia, associated to ${ }^{8}$

${ }^{67}$ Institut für Physik, Universität Rostock, Rostock, Germany, associated to ${ }^{12}$

68 National Research Centre Kurchatov Institute, Moscow, Russia, associated to ${ }^{32}$

69 National University of Science and Technology MISIS, Moscow, Russia, associated to ${ }^{32}$

70 National Research Tomsk Polytechnic University, Tomsk, Russia, associated to ${ }^{32}$

71 Instituto de Fisica Corpuscular, Centro Mixto Universidad de Valencia - CSIC, Valencia, Spain, associated to $^{38}$

72 Van Swinderen Institute, University of Groningen, Groningen, The Netherlands, associated to ${ }^{43}$

73 Los Alamos National Laboratory (LANL), Los Alamos, United States, associated to ${ }^{61}$

a Universidade Federal do Triângulo Mineiro (UFTM), Uberaba-MG, Brazil

${ }^{b}$ Laboratoire Leprince-Ringuet, Palaiseau, France

c P.N. Lebedev Physical Institute, Russian Academy of Science (LPI RAS), Moscow, Russia

d Università di Bari, Bari, Italy

e Università di Bologna, Bologna, Italy

$f$ Università di Cagliari, Cagliari, Italy

$g$ Università di Ferrara, Ferrara, Italy

$h$ Università di Genova, Genova, Italy

${ }^{i}$ Università di Milano Bicocca, Milano, Italy

$j$ Università di Roma Tor Vergata, Roma, Italy

* Università di Roma La Sapienza, Roma, Italy

${ }^{l}$ AGH - University of Science and Technology, Faculty of Computer Science, Electronics and Telecommunications, Kraków, Poland

$m$ LIFAELS, La Salle, Universitat Ramon Llull, Barcelona, Spain

$n$ Hanoi University of Science, Hanoi, Vietnam

- Università di Padova, Padova, Italy

$p$ Università di Pisa, Pisa, Italy

$q$ Università degli Studi di Milano, Milano, Italy

$r$ Università di Urbino, Urbino, Italy

$s$ Università della Basilicata, Potenza, Italy

${ }^{t}$ Scuola Normale Superiore, Pisa, Italy

u Università di Modena e Reggio Emilia, Modena, Italy

$v$ Iligan Institute of Technology (IIT), Iligan, Philippines

w Novosibirsk State University, Novosibirsk, Russia

$x$ National Research University Higher School of Economics, Moscow, Russia

$\dagger$ Deceased 